\title{
Genomic basis of endosymbiont-conferred protection against an insect parasitoid
}

\author{
Allison K. Hansen, ${ }^{1,4}$ Christoph Vorburger, ${ }^{2,3}$ and Nancy A. Moran ${ }^{1}$ \\ ${ }^{1}$ Department of Ecology and Evolutionary Biology, Yale University, West Haven, Connecticut 06516-7388, USA; ${ }^{2}$ Institute of Integrative \\ Biology, ETH Zürich, 8092 Zürich, Switzerland; ${ }^{3}$ EAWAG, Swiss Federal Institute of Aquatic Science and Technology, 8600 \\ Dübendorf, Switzerland
}

\begin{abstract}
Bacterial endosymbionts exert a variety of beneficial effects on insect hosts. In pea aphids (Acyrthosiphon pisum), several inherited endosymbiont species protect their hosts against parasitoid wasps, which are major natural enemies. However, strains of these symbiont species vary in their ability to confer protection against parasitoids, with some conferring almost complete protection and others conferring almost none. In this study, two strains of the endosymbiont Regiella insecticola ( $R$. insecticola 5.15 and $R$. insecticola LSR1) were found to differ in ability to protect pea aphids attacked by the parasitoid Aphidius ervi. Parasitism trials reveal that $R$. insecticola 5.15 , but not $R$. insecticola LSR1, significantly reduced parasitoid success and increased aphid survivorship. To address the potential genetic basis of protection conferred by $R$. insecticola 5.15 we sequenced the genome of this symbiont strain, and then compared its gene repertoire with that of the already sequenced nonprotective strain $R$. insecticola LSR1. We identified striking differences in gene sets related to eukaryote pathogenicity. The protective strain $R$. insecticola 5.15 encoded five categories of pathogenicity factors that were missing or inactivated in $R$. insecticola LSR1. These included genes encoding the O-antigen biosynthetic pathway, an intact Type 1 Secretion System and its secreted RTX toxins, an intact SPI-1 Type 3 Secretion System and its effectors, hemin transport, and the twocomponent system PhoPQ. These five pathogenicity factors and translocation systems are hypothesized to collectively play key roles in the endosymbiont's virulence against parasitoids, resulting in aphid protection. Mechanisms through which these factors may target parasitoids are discussed.
\end{abstract}

[Supplemental material is available for this article.]

Insects possess multiple modes of resistance against eukaryotic natural enemies (Vinson and Iwantsch 1980; Gross 1993). One insect defense against parasitoids and pathogens is the beneficial association with inherited bacterial endosymbionts (Oliver et al. 2003, 2005, 2009; Scarborough et al. 2005; Vorburger et al. 2010). A mechanistic understanding of endosymbiont-based resistance toward parasitoids is beginning to emerge, primarily due to genomic approaches (Moran et al. 2005; Degnan and Moran 2008; Degnan et al. 2009; Oliver et al. 2009). Endosymbionts known to confer resistance are currently unculturable, and thus, mutagenesis assays to identify putative pathogenicity factors are not yet possible.

The most well-understood model system in insect endosymbiont-parasitoid interactions involves the pea aphid (Acyrthosiphon pisum), its several facultative endosymbionts in the Enterobacteriaceae, and its dominant parasitoid natural enemy (Aphidius ervi). In A. pisum, some strains of the facultative endosymbiont species Serratia symbiotica and Hamiltonella defensa provide resistance against developing parasitoid larvae, independent of aphid genotype (Oliver et al. 2003, 2005). In contrast, a strain of the sister species of $H$. defensa, Regiella insecticola (str. 5AU), does not confer resistance to A. pisum (Oliver et al. 2003). In H. defensa, defense is dependent on a bacteriophage that codes for multiple toxin genes and lyses symbiont cells; this bacteriophage is called APSE (A. pisum secondary endosymbiont) (Moran et al. 2005; Degnan and Moran 2008; Degnan et al. 2009; Oliver et al. 2009).

In another aphid species, Myzus persicae, a strain of $R$. insecticola (str. 5.15), was found to cause near-complete resistance toward the

\footnotetext{
${ }^{4}$ Corresponding author.

E-mail allison.hansen@yale.edu.

Article published online before print. Article, supplemental material, and publication date are at http://www.genome.org/cgi/doi/10.1101/gr.125351.111.
}

parasitoid Aphidius colemani (Vorburger et al. 2010). Endosymbiontinduced resistance was confirmed by comparing naturally infected with cured $M$. persicae clones and by comparing uninfected with artificially infected clones in both M. persicae and also in Aphis fabae, a distantly related aphid species. Thus, resistance is associated with the presence of $R$. insecticola 5.15 and is not dependent on aphid genotype. However, the underlying mechanism is unknown.

In this study we address the potential genetic basis of the protection conferred by $R$. insecticola 5.15 . A previously sequenced strain, $R$. insecticola LSR1 (Degnan et al. 2010), is closely related to $R$. insecticola $5 \mathrm{AU}$, and both originated from A. pisum collected in New York, USA. Preliminary assays suggested that $R$. insecticola LSR1 resembled $R$. insecticola 5AU in failing to confer any resistance to $A$. ervi (NA Moran, unpubl.). To verify that $R$. insecticola LSR1 and $R$. insecticola 5.15 differ in protective ability against parasitoids, we tested both in the same host insect-parasitoid system, A. pisum and $A$. ervi. We sequenced the $R$. insecticola 5.15 genome and compared it with the genome of $R$. insecticola LSR1. Based on these results, we developed hypotheses for the basis of the protective phenotype observed in $R$. insecticola 5.15 .

\section{Results and Discussion}

\section{Parasitism trials}

For each of two A. pisum clones, 5A and LSR1, we established three sublines: $R$. insecticola 5.15-infected, $R$. insecticola LSR1-infected, and uninfected control. Parasitism resistance assays revealed no significant difference between the two A. pisum clones, but a significant effect of subline (Table 1). This effect is attributable to a defense conferred by $R$. insecticola 5.15 (Fig. 1A). In the $5 \mathrm{~A}$ aphids, infection by $R$. insecticola 5.15 lowered the rate of successful parasitism by 
Table 1. Analysis of deviance (proportion mummified) and analysis of variance (wasp development time) results for the parasitism trials

\begin{tabular}{|c|c|c|c|c|c|c|c|c|}
\hline \multirow[b]{2}{*}{ Effect } & \multicolumn{4}{|c|}{ Proportion mummified } & \multicolumn{4}{|c|}{ Development time } \\
\hline & df & Deviance & $F$ & $P$ & df & MS & $F$ & $P$ \\
\hline Block & 9 & 48.30 & 1.39 & 0.223 & 9 & 0.37 & 3.98 & 0.001 \\
\hline Aphid clone & 1 & 1.95 & 0.50 & 0.482 & 1 & 0.16 & 1.72 & 0.197 \\
\hline Subline & 2 & 35.28 & 4.57 & 0.016 & 2 & 0.31 & 3.37 & 0.044 \\
\hline Clone $\mathrm{x}$ subline & 2 & 16.27 & 2.11 & 0.134 & 2 & 0.23 & 2.53 & 0.091 \\
\hline Residual & 42 & 172.78 & & & 42 & 0.09 & & \\
\hline
\end{tabular}

about half, relative to uninfected or R. insecticola LRS1-infected lines. The effect appeared to be less in the LSR1 background; however, the clone $\mathrm{x}$ symbiont interaction was not significant (Table 1). Consistent with a negative effect of $R$. insecticola 5.15 on parasitoid development, the parasitoids that did develop to pupation in this line had a slower developmental rate as compared with those developing in R. insecticola LSR1 or uninfected lines (Fig. 1B). This was reflected in a significant subline effect in the analysis (Table 1 ).

\section{Genome sequencing and annotation}

The 454 Life Sciences (Roche) sequencing run yielded 580,245 reads that totaled $222.91 \mathrm{Mbp}$, and assembled into 6665 contigs, corresponding to genome sequences of $A$. pisum, Buchnera, and $R$. insecticola. No lytic or lysogenic phage was present, based on both genomic sequences or PCR screening evidence. We identified 562 unique contigs comprising 2.01 Mbp assignable to $R$. insecticola-5.15 (Table 2). Several indicators reveal that the $R$. insecticola 5.15 genome sequence is near completion. First, the genome size of $R$. insecticola 5.15 (at $2.01 \mathrm{Mbp}$ ) is similar to the previously sequenced $R$. insecticola LSR1 (at 2.07 Mbp) (Degnan et al. 2010; Table 2). Second, the retrieved contigs included 186 of 205 single-copy genes (SICO) that are present in most Gammaproteobacteria (Lerat et al. 2003). Third, an average read coverage of $16.3 \times$ was achieved for contigs containing these genes. Assuming reads follow a Poisson distribution, $>99.99 \%$ of the genome should be covered (Lander and Waterman 1988). Thus, pathogenicity factors and related metabolisms can be confidently identified for $R$. insecticola 5.15 .

\section{Comparing Regiella genomes}

Based on phylogenetic analyses of known $R$. insecticola strains, $R$. insecticola 5.15 appears to be somewhat divergent from $R$. insecticola LSR1 and other strains originating from A. pisum (Fig. 2). Estimates of nonsynonymous substitutions per nonsynonymous site $\left(d_{\mathrm{N}}\right)$ and synonymous substitutions per synonymous site $\left(d_{\mathrm{S}}\right)$ were calculated for each SICO ortholog pair between $R$. insecticola LSR1 and $R$. insecticola $5.15(n=184)$. The mean $d_{\mathrm{N}}$ is $0.0173 \pm 0.05 \mathrm{SD}$, the mean $d_{\mathrm{S}}$ is $0.090 \pm 0.25 \mathrm{SD}$, while the mean $d_{\mathrm{N}} / d_{\mathrm{S}}$ ratio is $0.225 \pm 0.32 \mathrm{SD}$, indicating purifying selection on these core genes. These low divergence levels are consistent with classification of the strains as a single bacterial species. For comparison, mean $d_{\mathrm{N}}$ and $d_{\mathrm{S}}$ are much higher between sister species $R$. insecticola LSR1 and $H$. defensa $\left(d_{\mathrm{N}}=0.26, d_{\mathrm{S}}>3.0\right)$ (Degnan et al. 2010).

Using JGI's phylogenetic profiler and BLASTP, we identified 1671 genes in R. insecticola 5.15 that had homologs in R. insecticola LSR1, and 1461 genes in $R$. insecticola LSR1 that had homologs in $R$. insecticola 5.15 (Fig. 3). The larger number in R. insecticola 5.15 is due to two causes. First, $75.3 \%$ of multiple copies in R. insecticola
5.15 relative to $R$. insecticola LSR1 are due to mobile genes such as transposases, ISR elements, and repetitive hypothetical proteins, indicating greater numbers of these repetitive genes in $R$. insecticola 5.15 . Second, $24.7 \%$ of genes with more copies in $R$. insecticola 5.15 involve gene fragments that are either on the edge of contigs, with truncations, or that contain premature stop codons resulting in multiple open reading frames per gene. These likely represent true pseudogenes, since such fragmentation was not found to occur in essential genes.

We also identified 642 unique genes present in $R$. insecticola 5.15 with no homologs in $R$. insecticola LSR1 (Fig. 3; Supplemental Table 1). Of these R. insecticola 5.15-specific genes, 32.7\% are hypothetical, with no significant homology with known proteins (Suppleental Table 1), whereas the remaining 67.3\% had significant BLASTP hits and/or belonged to several functional gene categories (e.g., COG clusters, Pfam; see Methods) (Fig. 3; Supplemental Tables 1, 2). The large number of hypothetical proteins may partially be due to nonintact genes, since $48 \%$ of hypothetical proteins were on the edge of contigs, as expected when ORFs are interrupted by IS elements. In order to verify the status of genes unique to 5.15 , we carried out PCR amplification and Sanger sequencing for apparent pseudogenes present only in 5.15 (2.2\% of genes). We checked all apparent pseudogenes within contigs for cases in which two ORFs for regions of the same genes were adjacent and for cases in which a truncated gene was flanked by a hypothetical gene on the same

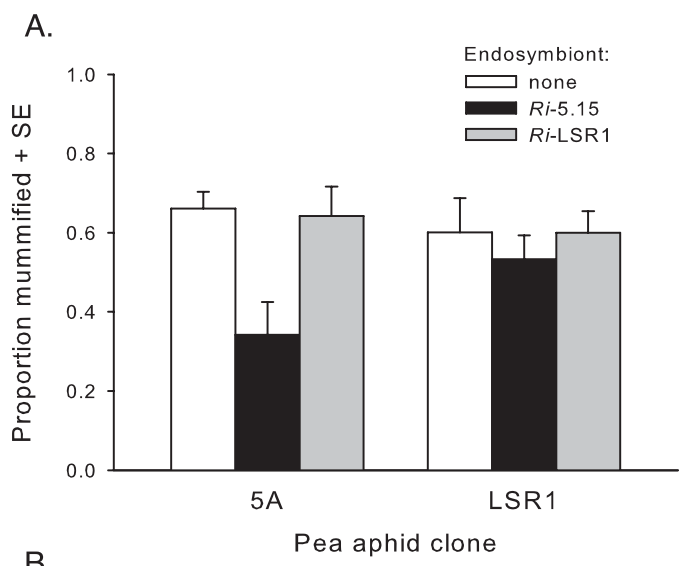

B.

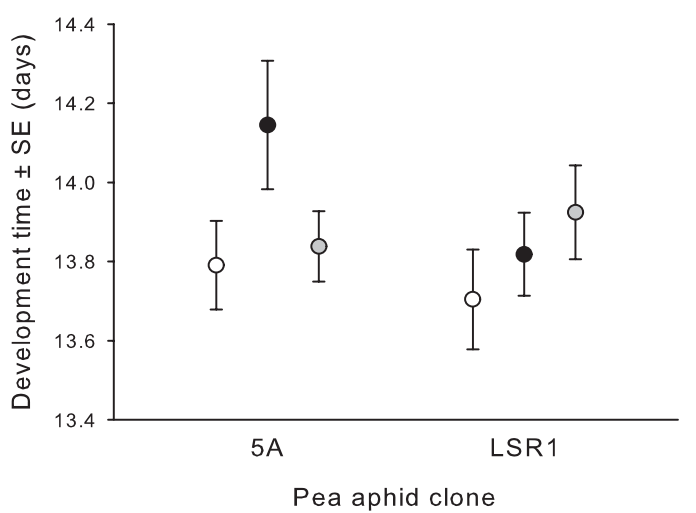

Figure 1. Effects of $R$. insecticola strains on development of $A$. ervi in two clones of $A$. pisum, 5A and LSR1. (A) Proportion of parasitized wasps developing successfully in each aphid line. (B) Developmental time (days from oviposition to emergence as adults) in wasps that did develop successfully in each aphid line. Values are means $+/-$ SE. 
Table 2. Genome comparison statistics of Regiella insecticola 5.15 with other sequenced insect-associated Enterobacteriaceae

\begin{tabular}{lccccc}
\hline & $\begin{array}{c}\text { Regiella } \\
\text { insecticola } \\
\text { R.5.15 }\end{array}$ & $\begin{array}{c}\text { Regiella } \\
\text { insecticola } \\
\text { LSR1 }\end{array}$ & $\begin{array}{c}\text { Hamiltonella } \\
\text { defensa } \\
\text { 5AT }\end{array}$ & $\begin{array}{c}\text { Serratia } \\
\text { symbiotica } \\
\text { TUC }\end{array}$ & $\begin{array}{c}\text { Photorhabdus } \\
\text { luminescens } \\
\text { laumondiiTTO1 }\end{array}$ \\
\hline Chromosome size (bp) & $2,013,072$ & $2,067,400$ & $2,169,363$ & $2,789,218$ & $5,688,987$ \\
G+C\% & $42.6 \%$ & $42.5 \%$ & $40.5 \%$ & $52 \%$ & $42.8 \%$ \\
Protein-coding genes & 2313 & 1769 & 2158 & 2098 & 4895 \\
tRNA & 34 & 37 & 43 & 44 & 85 \\
16S rRNA & 2 & 4 & 3 & 5 & 7 \\
\hline
\end{tabular}

${ }^{a}$ Minimal number of expected $16 \mathrm{~S}$ rRNA genes.

strand (Supplemental Methods). Eight of 15 putative pseudogenes possessed errors, all in homopolymers, and these contigs were corrected.

Of the genes distinctive to $R$. insecticola $5.15,39 \%$ had functional information, and these belonged to 19 COG categories (Fig. 3; Supplemental Table 2). Genes present in $R$. insecticola 5.15 and not $R$. insecticola LSR1 include unknown conserved bacterial genes, transposases, Type I, III, and IV Secretion System components, iron transporters, signal transduction systems, and phage-related proteins (Supplemental Table 2). Of the R. insecticola 5.15-specific genes, 34 encoded factors linked to eukaryote pathogenicity (Table 3). Most genes encoding pathogenicity factors were intact, with lengths of $>90 \%$ of their best blastx hit homolog (Table 3). Since 454 sequencing can miscall runs of a single base, any genes with homopolymers of more than $5 \mathrm{nt}$ were Sanger sequenced to confirm that errors were not present. Only one gene $(\mathrm{prgH})$ possessed a homopolymer error introducing a premature stop codon, and this contig was corrected.

\section{Unique pathogenicity-related factors and translocation systems}

\section{Endotoxins}

The outer membrane of the Gram-negative bacterial cell wall is composed of lipopolysaccharide (LPS), consisting of Lipid A, a core oligopolysaccharide, and an $\mathrm{O}$-antigen polysaccharide. LPS is referred to as an endotoxin and is associated with virulence. In particular, the O-antigen polysaccharide is important for host virulence since it is known to play roles in host cell adhesion (Jacques 1996; Jacques and Paradis 1998; Edwards et al. 2000), resistance to phagocytes (Liang-Takasaki et al. 1982), and resistance to host immune responses and surveillance (Liang-Takasaki et al. 1983; Jimenez-Lucho et al. 1987; Moran et al. 1996; Rietschel et al. 1996). Both $R$. insecticola strains possess similar gene sets with respect to biosynthesis of Lipid A and the core polysaccharide. In contrast, gene sets underlying biosynthesis of the O-antigen polysaccharide are drastically different between strains. $R$. insecticola 5.15 encodes all genes required for $\mathrm{O}$-antigen polysaccharide biosynthesis, whereas the entire subpathway for dtDP-alphaL-rhamnose ( $r f b A B C D$ cluster) is missing from the genome of $R$. insecticola LSR1 (Table 3). As a result, the O-antigen polysaccharide is predicted to be completely absent or highly divergent in $R$. insecticola LSR1 (i.e., rough LPS) as compared with $R$. insecticola 5.15. A phylogenetic analysis of $\mathrm{O}$-antigen proteins from R. insecticola 5.15 and other Proteobacteria indicates that the copy in $R$. insecticola may have arisen through horizontal gene transfer, as it is distantly related to the copies in other Enterobacteriaceae and more closely related to those in more distant Gammaproteobacteria and in Betaproteobacteria (Supplemental Fig. 1).
Exotoxins and Type I secretion systems (TISS)

RTX (Repeats-in-Toxins)-related toxins are the most abundant group of exotoxins encoded in the genome of $R$. insecticola 5.15 (Table 4), as well as in genomes of both $H$. defensa $5 \mathrm{AT}$ and R. insecticola LSR1 (Degnan et al. 2009, 2010). RTX toxins are exotoxins secreted by the T1SS into the extracellular environment (Holland et al. 2005) that are associated with cytotoxicity and pathogenesis toward eukaryotes (Coote 1992). The genome of $R$. insecticola 5.15 encodes eight putative proteins with significant NCBI Conserved Domain RPSBLAST hits to RTX toxins and related $\mathrm{Ca}^{2+}$-binding proteins (COG2931). In H. defensa and both $R$. insecticola strains, putative RTX (COG2931) genes also encode cysteine protease effector domains toward the $\mathrm{N}$ terminus for both $\mathrm{C} 80$ and M10 families, as in Yersinia pseudotuberculosis (Sheahan et al. 2007). R. insecticola 5.15 encodes several RTX toxins with homologs present in $H$. defensa or $R$. insecticola LSR1 (Table 4). One large putative RTX toxin (2262 amino acids, Rin_00023541) in R. insecticola 5.15 lacks homologs in either $H$. defensa or $R$. insecticola and encodes multiple serine protease domains, a Lysin domain, and COG2931 (Table 3).

Putative RTX toxins may be functional in R. insecticola 5.15, since the genome encodes an intact T1SS operon, and TolC, an intact outer membrane protein (Rin_00000710). In contrast, $R$. insecticola LSR1 lacks an intact T1SS (Degnan et al. 2010). Screens of multiple $H$. defensa and $R$. insecticola strains revealed that the T1SS is intact in most $H$. defensa strains, but not in any $R$. insecticola strains, with the exception of $R$. insecticola 5.15 (Fig. 4) (accession nos. JF928377JF928387). During attempts to amplify T1SS genes, only small fragments were obtained in the majority of $R$. insecticola strains, suggesting that genes encoding the T1SS are inactivated and fragmentary as in $R$. insecticola LSR1. Thus, the T1SS is uniquely present in $R$. insecticola 5.15 among sampled $R$. insecticola strains, including LSR1. The T1SS and its associated exotoxins, such as RTX toxins, are candidates for protection against parasitoids conferred by R. insecticola 5.15 (Fig. 5).

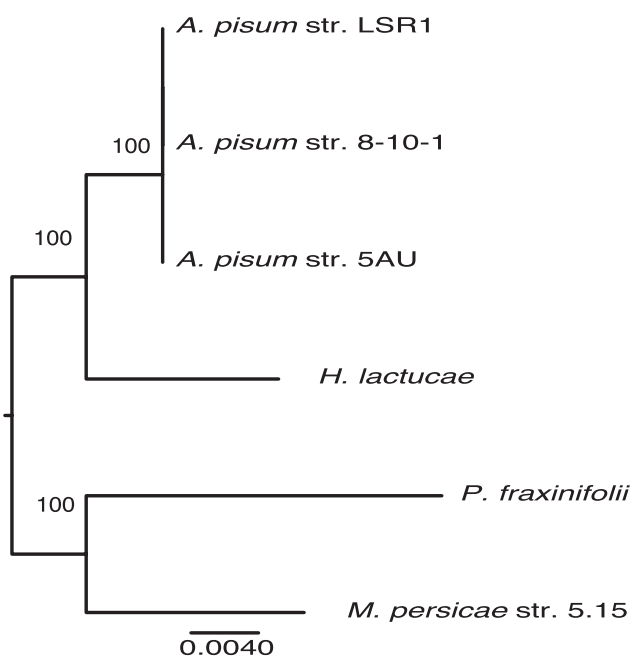

Figure 2. Phylogenetic relationships of $R$. insecticola strains based on a 1977-bp alignment from three concatenated core genes ( $a c c D$, gyrB, and murE) using RAxML with 100 bootstraps. Aphid host names are used to label branches. 


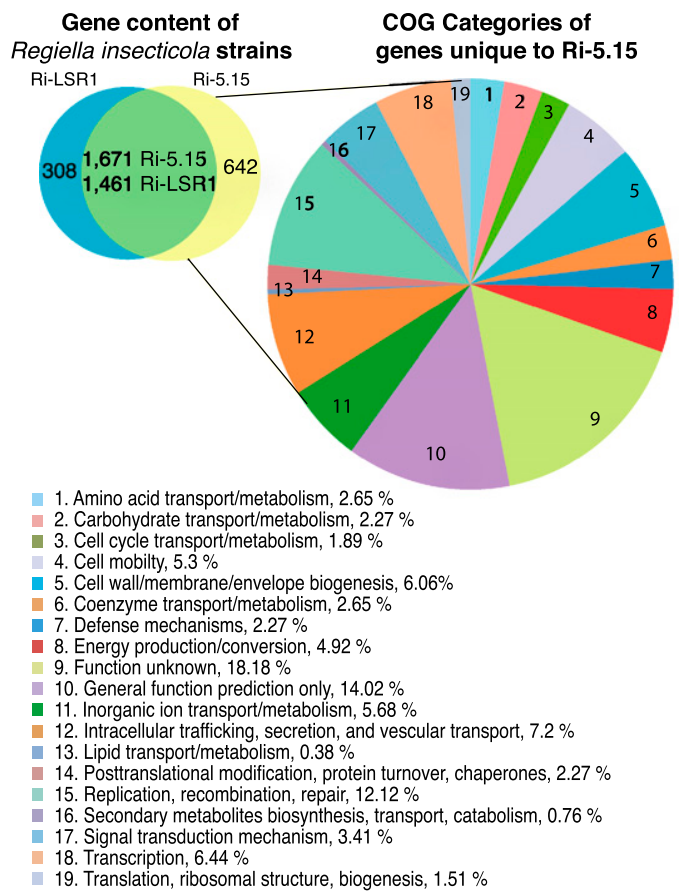

Figure 3. Comparative genomics of $R$. insecticola LSR1 and $R$. insecticola 5.15. The left pie represents the number of genes homologous and unique to each $R$. insecticola strain. The right pie represents the relative proportion of novel $R$. insecticola 5.15 genes in 19 COG categories.

Genes encoding several other exotoxins were identified in the genome of $R$. insecticola 5.15 (Table 4). But, in contrast to the RTXrelated toxins, most of these toxins appear to be nonfunctional, and thus not important in virulence toward parasitoids. Several of these toxins are on the ends of contigs, however, and may still be intact. A gene encoding the putative hemolysin toxin (PhlA), belonging to a two-partner secretion system was identified; however, a gene encoding the secretion protein PhlB, required for exotoxin secretion into the extracellular environment (Brillard et al. 2002), was not detectable. Also, R. insecticola 5.15 encoded three truncated toxins belonging to the clostridial glycosylating toxin family and a truncated calmodulin-sensitive adenylate cyclase toxin. Another toxin encoded in $R$. insecticola 5.15 that is related to insect pathogenicity is makes caterpillars floppy (Mcf) (Daborn et al. 2002), for which six divergent homologs were detected, including some that are distant from homologs present in R. insecticola LSR1. One Mcf protein (Rin_00019530), located on the end of a contig, also encodes a TcdA/TcdB pore-forming Pfam domain. Another eukaryotic toxin found in $R$. insecticola 5.15 is a 36 -amino acid putative YD-repeat toxin. This toxin is also found in $R$. insecticola LSR1 and appears to be truncated in both symbionts.

\section{Type III secretion system (T3SS) and effectors}

The T3SS and its associated effectors are important for many interactions between Gram-negative bacteria and eukaryotic hosts, such as attachment and invasion of host cells, the avoidance of phagocytosis, and suppression of host cell innate immunity (Hueck 1998; Waterman and Holden 2003; Matsumoto and Young 2009). As in $H$. defensa, R. insecticola 5.15 encodes two types of T3SS (SPI-1 and SPI-2); in contrast, $R$. insecticola LSR1 only encodes SPI-2 (Table $3)$. Several putative T3SS effectors, such as the SPI-1 effector YopJ/P, were present in $R$. insecticola 5.15 and not in $R$. insecticola LSR1 (Table
3). In Yersinia this effector is responsible for inducing apotosis and suppressing the innate immune system by targeting MAPK and NF$\kappa \mathrm{B}$ signaling pathways (Orth et al. 2000). Because the SPI-1 T3SS and associated effectors are present in $R$. insecticola 5.15 and not in $R$. insecticola LSR1, these factors are candidates for genes underlying virulence toward parasitoids (Fig. 5).

\section{Two component systems}

$R$. insecticola 5.15 encodes PhoPQ, a two-component signaling system that is associated with virulence in Salmonella sp., Shigella sp., Pseudomonas aeruginosa, Mycobacterium tuberculosis, Neisseria meningitidis, Y. pseudotuberculosis, and Yersinia pestis. Both R. insecticola LSR1 and the sister species, $H$. defensa, lack phoPQ. Phylogenetic analyses of the $R$. insecticola 5.15 phoPQ operon show that it is closest to homologs in other Enterobacteriaceae (Supplemental Figs. 2-4), an observation that is consistent with vertical transmission of phoPQ and loss in both $H$. defensa and LSR1, or with the horizontal acquisition by $R$. insecticola 5.15 from Yersinia or a relative.

Broadly, PhoPQ-induced phenotypes are associated with bacterial cell envelope modifications, which specifically include increased survival in macrophages, invasion of cells, tolerance to acidic $\mathrm{pH}$ and antimicrobial peptides, resistance to antibiotics, modification of Lipid A, and antigen presentation (Miller et al. 1989; Groisman et al. 1997; Guo et al. 1997; Ernst et al. 1999; Macfarlane et al. 1999; Moss et al. 2000; Oyston et al. 2000; Johnson et al. 2001; Groisman 2001; Perez et al. 2001; Grabenstein et al. 2004). This two-component system controls expression of the pbgPE operon in Photorhabdus luminescens and is associated with virulence against insects; pbgPE mutants are lacking an O-antigen, resulting in increased sensitivity to insect-produced antimicrobial peptides and acidic pH (Derzelle et al. 2004; Bennett and Clarke 2005).

In addition, the PhoPQ-activated, pathogenicity-related protein PqaA (Table 3 ) is encoded in the genome of $R$. insecticola 5.15, but absent from the genome of $R$. insecticola LSR1. Interestingly, this protein is active against the antimicrobial peptide melittin (Baker et al. 1997), found in bee and parasitoid venom (Habermann 1972; Uçkan et al. 2004). Potentially PhoPQ and PqaA play a key role in virulence toward parasitoids, possibly through PqaA-induced resistance to parasitoid venom-associated antimicrobial peptides, under PhoPQ regulation.

\section{Iron acquisition}

Availability of iron to bacteria living within host environments is generally limited; successful uptake and maintenance of iron is essential for bacterial growth and virulence (Thompson et al. 1999; Anzaldi and Skaar 2010; Watson et al. 2010; Braun and Hantke 2011). Bacterial pathogens generally possess multiple iron transport systems, depending on what type of iron is available in the host environment (Braun and Hantke 2011). In P. luminescens, ironuptake via Yfe- and TonB-dependent transporters is required to induce virulence against insect larvae (Watson et al. 2010). The genome of $R$. insecticola 5.15 encodes multiple iron and hemin transport systems, such as the $\mathrm{ABC}$ iron transporter Yfe, the non-ABC iron transporter Feo, and the TonB-dependent hemin transporter Hmu. $R$. insecticola LSR1 encodes transporters Yfe and Feo, but lacks the TonB-dependent Hmu operon (Table 3). In turn, $R$. insecticola 5.15 encodes a diverse array of iron transporters relative to $R$. insecticola LSR1; potentially $R$. insecticola requires iron transporters to induce parasitoid virulence gene sets (Fig. 5). Alternatively, because $R$. insecticola 5.15 possesses a broader array of iron transporters it may be more successful than $R$. insecticola LSR1 in competing with the 
Table 3. Gene sets of pathogenicity-related factors found in Regiella insecticola R5.15, but absent in $R$. insecticola LSR 1

\begin{tabular}{|c|c|c|c|c|c|}
\hline Gene ID & Genename & BLASTP best hit & $\%$ Similarity & Coverage & NCBI BLAST ID \\
\hline Rin_00002460 & $r f b A$ & Polaromonas sp. JS666 & 79.5 & $99 \%$ & YP_550810.1 \\
\hline Rin_00002480 & $r f b B$ & Dechloromonas_efense_RCB & 73.6 & $99 \%$ & YP_284463.1 \\
\hline Rin_00002450 & $r f b c$ & Nitrosomonas europaea ATCC 19718 & 72.4 & $99 \%$ & NP_840757.1 \\
\hline Rin_00002470 & $r f b D$ & Achromobacter piechaudii ATCC 43553 & 60.5 & $97 \%$ & ZP_06684951.1 \\
\hline Rin_00021640 & $r t \times B 1$ & H. defensa 5AT fused ATP binding/permease domains & 75.5 & $99 \%$ & YP_002923191.1 \\
\hline Rin_00021630 & $r t \times D$ & H. defensa 5AT & 70.1 & $99 \%$ & YP_002923190.1 \\
\hline Rin_00021620 & $r t \times B 2$ & H. defensa 5AT & 78.4 & $96 \%$ & YP_002923189.1 \\
\hline Rin_00023541 & RTX toxin & Actinobacillus minor NM305 & 48 & $83 \%$ & ZP_04752633.1 \\
\hline Rin_00010760 & $\operatorname{prgk}$ & H. defensa 5AT & 66.7 & $83 \%$ & YP_002924023.1 \\
\hline Rin_00000900 & prgl & Shigella flexneri 2002017 & 40 & $57 \%$ & NP_085300.1 \\
\hline Rin_00022530 & $\mathrm{prgH}$ & H. defensa 5AT & 48.4 & $92 \%$ & YP_002924026.1 \\
\hline Rin_00022520 & $\operatorname{lag} B$ & Salmonella enterica arizonae sv 62:z4,z23 RSK2980 & 60.9 & $91 \%$ & YP_001569191.1 \\
\hline Rin_00020660 & spam & H. defensa 5AT & 59.6 & $91 \%$ & YP_002924274.1 \\
\hline Rin_00020670 & $\operatorname{span}$ & Salmonella enterica & 37 & $41 \%$ & gb|AAC45010.1 \\
\hline Rin_00020680 & spaO & H. defensa 5AT & 41.9 & $93 \%$ & YP_002924277.1 \\
\hline Rin_00020690 & spap & H. defensa 5AT & 78.5 & $99 \%$ & YP_002924278.1 \\
\hline Rin_00020700 & spaQ & H. defensa 5AT & 80.2 & $98 \%$ & YP_002924279.1 \\
\hline Rin_00009770 & inva & H. defensa 5AT & 79.9 & $99 \%$ & YP_002924271.1 \\
\hline Rin_00009780 & $i n v B$ & H. defensa 5AT & 37 & $97 \%$ & YP_002924272.1 \\
\hline Rin_00009790 & $\operatorname{inv} C$ & H. defensa 5AT & 70 & $99 \%$ & YP_002924273.1 \\
\hline Rin_00010820 & уор/P & Vibrio parahaemolyticus & 33 & $76 \%$ & gb|AAT08443.1 \\
\hline Rin_00001220 & SopD & Yersinia mollaretii ATCC 43969 & 41.1 & $51 \%$ & ZP_04641767.1 \\
\hline $\operatorname{Rin} 00005600$ & Ipa & Escherichia coli 53638 & 38.1 & $96 \%$ & ZP_03002040.1 \\
\hline Rin_00005610 & Ipa & Shigella boydii CDC 3083-94 & 39 & $66 \%$ & YP_001883129.1 \\
\hline $\operatorname{Rin} 00009820$ & phop & Yersinia aldovae ATCC 35236 & 86.2 & $99 \%$ & ZP 04619595.1 \\
\hline Rin_00009830 & phoQ & Yersinia enterocolitica 8081 & 80.3 & $99 \%$ & YP_001006003.1 \\
\hline Rin_00006990 & $p q a A$ & Yersinia intermedia ATCC 29909 & 53.7 & $99 \%$ & ZP_04638247.1 \\
\hline Rin_00016410 & hmuR & Yersinia ruckeri ATCC 29473 & 62.6 & $97 \%$ & ZP_04615019.1 \\
\hline Rin_00019020 & hmuV & Serratia proteamaculans 568 & 65.2 & $94 \%$ & YP_001478394.1 \\
\hline Rin_00019030 & hmuU & Yersinia rohdei ATCC 43380 & 72 & $90 \%$ & ZP_04613865 \\
\hline Rin_00019040 & hmuT & Yersinia ruckeri ATCC 29473 & 68.7 & $93 \%$ & ZP_04615021.1 \\
\hline Rin_00019050 & hmuS & Yersinia ruckeri ATCC 29473 & 61.5 & $98 \%$ & ZP_04615020.1 \\
\hline Rin_00019060 & hmus & Serratia proteamaculans 568 & 50.6 & $74 \%$ & YP_001478397.1 \\
\hline Rin_00019070 & hmuR & Yersinia ruckeri ATCC 29473 & 63.7 & $99 \%$ & ZP_04615019.1 \\
\hline
\end{tabular}

parasitoid for iron. Iron deficiency in parasitized aphids has been shown to result in arrested development of parasitoids (Zohdy 1976). Thus, bacterial competition for iron may slow wasp development, or may be lethal for the wasp.

\section{Amino acid biosynthesis and metabolism}

Larval endophagous parasitoids of aphids, such as A. ervi, feed on insect host haemolymph via ingestion and trans-cuticle absorption (Pennacchio et al. 1995; Rahbé et al. 2002; Giordana et al. 2003; Caccia et al. 2005). As in animals, generally parasitoids do not encode pathways for essential amino acid biosynthesis and must obtain these amino acids from their diet, particularly in the form of free amino acids during early larval development (Thompson 1986). In A. ervi, external sources of essential and nonessential free amino acids play a substantial role in early larval nutrition (Giordana et al. 2003; Caccia et al. 2005). Because an adequate supply of free amino acids in aphid haemolymph is required for successful $A$. ervi development and survival (Pennacchio et al. 1999; Rahbé et al. 2002), competition for free amino acids by $R$. insecticola 5.15 is a potential factor affecting parasitoid development. However, both $R$. insecticola strains encode similar gene sets for amino acid metabolisms and transport. Both have lost all essential amino acid biosynthetic pathways and have retained genes for corresponding active transporters. The only difference in amino acid biosynthetic abilities is that $R$. insecticola LSR1 and not $R$. insecticola 5.15 retains the pathway to synthesize threonine from aspartate. The overall similarity of amino acid metabolisms suggests that competition for limiting amino acids is not a basis of the defense conferred by R. insecticola 5.15.

\section{Conclusions}

Facultative insect endosymbionts are not essential for host survival and reproduction, but have been found to increase host fitness under particular conditions, such as attack by natural enemies (Oliver et al. 2003, 2005, 2009; Scarborough et al. 2005; Vorburger et al. 2010). Because most endosymbionts cannot be cultured axenically, genomic approaches are the primary means for elucidating the genetic mechanisms responsible for these mutualistic effects. Through comparative genomics and parasitism assays, we found a link between the symbiont-conferred protection against a wasp natural enemy and the presence of symbiont genes homologous to virulence factors known from pathogenic bacterial species. Thus, genes that underlie pathogenicity in other bacterial species are hypothesized to contribute to the observed beneficial effects of $R$. insecticola 5.15 on its aphid hosts, through differential effects on the wasp parasitoids.

A previous study found that $R$. insecticola 5.15 confers protection against the parasitoid species $A$. colemani in multiple aphid strains of M. persicae and in the aphid species Aphis fabae (Vorburger et al. 2010). We found that $R$. insecticola 5.15 also confers the aphid species A. pisum with protection against $A$. ervi by reducing wasp survivorship and development (Fig. 1). This ability to confer protection contrasts with previous studies on other strains of $R$. insecticola originating from $A$. pisum hosts, including $R$. insecticola LSR1. Collectively, these results suggest that $R$. insecticola 5.15 induces a generalized protective phenotype against Aphidius parasitoids in divergent aphid host species. Whether or not $R$. insecticola 5.15-infected aphids have lower fitness relative to uninfected aphids in an environment without parasitism pressure has yet to be tested. Aphids harboring $H$. defensa have lower

\section{Genome Research}


Table 4. Putative toxin genes found in Regiella insecticola 5.15

\begin{tabular}{|c|c|c|c|c|c|}
\hline Gene ID & Putative toxin & BLASTP best hit & $\%$ Similarity & Length (bp) & Gene ID \\
\hline Rin_00005680 & RTX (COG2931) & R. insecticola LSR1 & 88 & 5472 & ZP_07395317.1 \\
\hline Rin_00020460 & RTX (COG2931) & R. insecticola LSR1 & 52 & 4929 & ZP_07395793.1 \\
\hline Rin_00022590 & RTX (COG2931) & R. insecticola LSR1 & 37 & 5091 & ZP_07395875.1 \\
\hline Rin_00004730 & RTX (COG2931) & H. defensa 5AT & 57 & 6399 & YP_002924052.1 \\
\hline Rin_00010920 & RTX (COG2931) & R. insecticola LSR1 & 39 & 2448 & ZP_07395533.1 \\
\hline Rin_00012520 & RTX (COG2931) & R. insecticola LSR1 & 93 & 5583 & ZP_07396220.1 \\
\hline Rin_00008900 & RTX (COG2931) & R. insecticola LSR1 & 93 & 8142 & ZP_07395875.1 \\
\hline Rin_00023541 & RTX (COG2931) & Actinobacillus minor NM305 & 48 & 6789 & ZP_04752633.1 \\
\hline Rin_00004940 & Mcf & R. insecticola LSR1 & 50 & 2637 & ZP_07395018.1 \\
\hline Rin_00007680 & Mcf & Pseudomonas fluorescens & 55 & 339 & gbĪABY91230.1 \\
\hline Rin_00016680 & Mcf & $\begin{array}{l}\text { Aeromonas hydrophila subsp. } \\
\text { hydrophila ATCC } 7966\end{array}$ & 52.5 & 2142 & YP_855898.1 \\
\hline Rin_00007670 & Mcf & Photorhabdus luminescens & 28 & 687 & gblAAM88787.1 \\
\hline Rin_00010230 & Mcf & R. insecticola LSR1 & 49 & 1266 & ZP_07394566.1 \\
\hline Rin_00019530 & Mcf & R. insecticola LSR1 & 28 & 3117 & ZP_07394566.1 \\
\hline Rin_00004780 & Hemolysin A & Serratia odorifera $4 \mathrm{R} \times 13$ & 59 & 528 & ZP_06191388.1 \\
\hline Rin_00005100 & YD-repeat & R. insecticola LSR1 & 89 & 108 & ZP_07394992.1 \\
\hline Rin_00010940 & Cytotoxin L/B & R. insecticola LSR1 & 67 & 918 & ZP_07395432.1 \\
\hline Rin_00019510 & Toxin A & R. insecticola LSR1 & 90 & 288 & ZP_07395607.1 \\
\hline Rin_00019520 & Toxin B & R. insecticola LSR1 & 88 & 1413 & ZP_07395607.1 \\
\hline Rin_00008630 & $\begin{array}{l}\text { Calmodulin-sensitive } \\
\text { adenylate cyclase }\end{array}$ & R. insecticola LSR1 & 91 & 558 & ZP_07395590.1 \\
\hline
\end{tabular}

fitness relative to uninfected aphids (Oliver et al. 2008; Vorburger and Gouskov 2011).

The mechanism of $H$. defensa-induced protection against $A$. ervi parasitoids is dependent on the association of $H$. defensa with the lysogenic bacteriophage APSE (Oliver et al. 2009). In this study, no lytic or lysogenic phage is present in $R$. insecticola 5.15 , based on sequencing or PCR screens using degenerate primers for APSE sequences. Thus, the mechanisms of endosymbiont-induced resistance toward parasitoids differ between $H$. defensa and $R$. insecticola 5.15 .

By comparing genomes of protective and nonprotective $R$. insecticola strains, we found that numerous pathogenicity-related factors and translocation systems are unique to the protective strain 5.15. In particular, $R$. insecticola 5.15 protection may reflect the presence of five key eukaryotic pathogencity-related factors absent from the nonprotective strain, $R$. insecticola LSR1 (Fig. 5). These virulence gene sets are associated with the presence of an O-antigen, an intact T1SS and its secreted RTX toxins, an intact T3SS and its effectors, hemin transport, and the two-component system PhoPQ and related genes (Fig. 5).

One unresolved question is why these candidate "eukaryotic" pathogenicity factors would selectively target and kill the parasitoid wasp and not the aphid host. One difference between the parasitoid and aphid is the mode of $R$. insecticola 5.15 infection. In aphids, facultative endosymbionts, such as $R$. insecticola 5.15 , are vertically transmitted from mother to progeny during development of eggs or embryos. If $R$. insecticola 5.15 directly infects the wasp larva, then it must invade orally through the gut and/or through its cuticle. Different cell types and modes of bacterial infection can result in differential regulation of pathogenicity-related factors, as known for O-antigen adhesions that recognize and attach to specific eukaryotic cells (Jacques 1996), the SPI-1 T3SS that is important for invading certain host cells (Main-Hester et al. 2008), and signal-transduction systems such as PhoP-PhoQ (Groisman and Mouslim 2006). One way that bacterial pathogens can achieve virulence is by disabling, resisting, or interfering with their host's innate immune system via T3SS effectors such as YopJ and/or O-antigens. $R$. insecticola 5.15 may interact differentially with the wasp and aphid immune systems. For example, hymenopteran genomes, as represented by the parasitoid
Nasonia vitripennis, possess different gene sets in the Gram-negative activated immune deficiency (IMD) pathway compared with aphids (Gerardo et al. 2010) (BLASTP of Nasonia in NCBI, NZ_AAZX00000000). Also, exotoxins such as RTX toxins can be very specific for a particular cell type and organism (Coote 1992).

In summary, several eukaryote-targeted pathogenicity factors are implicated as the basis for the ability of R. insecticola 5.15 to kill, or slow development of parasitoid wasps, resulting in protection of the aphid host.

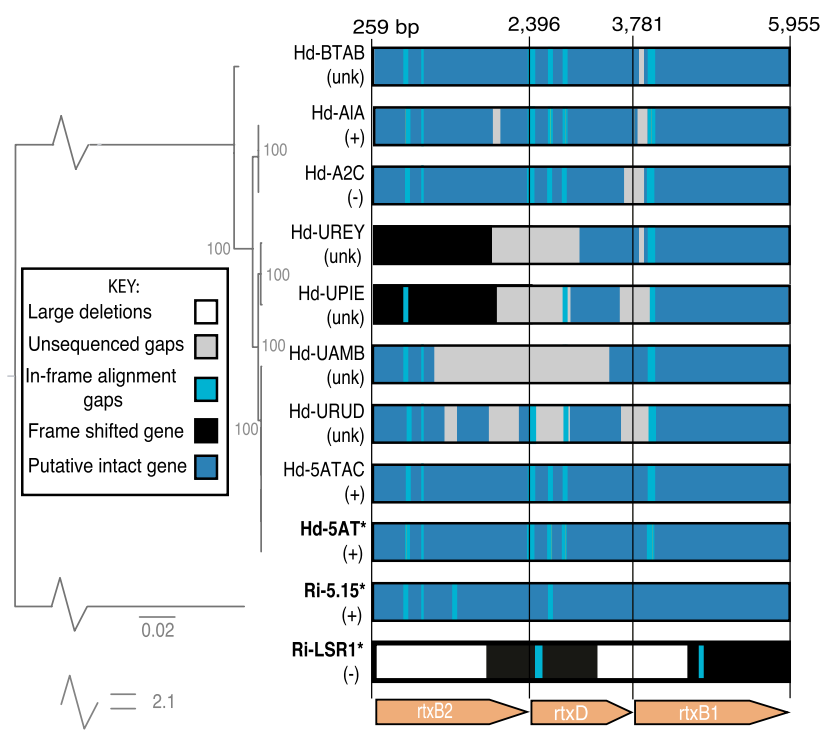

Figure 4. T1SS nucleotide gene alignments of $H$. defensa and R. insecticola strains in reference to $H$. defensa 5AT. Bolded strains with stars represent sequenced genomes. Phylogenetic relationships of strains are based on a 4334-bp alignment from T1SS genes (rtxB2, rtsD, rtxB1) using RAxML with 100 bootstraps. Plus signs indicate that the strain protects its aphid host against parasitoids, minus signs indicate a nonprotective phenotype, and "unk" indicates unknown phenotype, based on previous studies (Oliver et al. 2003, 2009; Vorburger et al. 2010) and this study. 


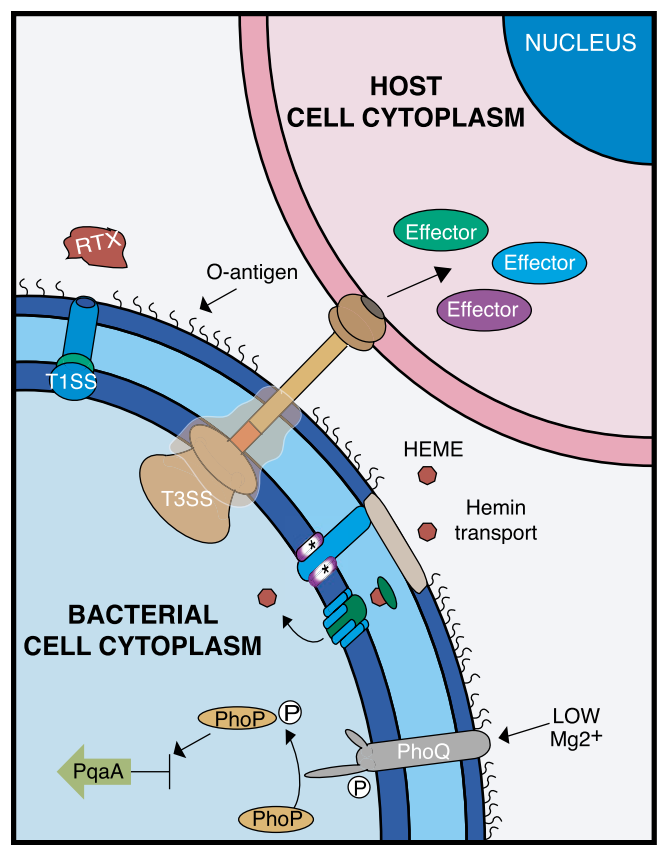

Figure 5. Eukaryotic virulence factors present in the protective endosymbiont strain $R$. insecticola 5.15 , but absent in the nonprotective strain $R$. insecticola LSR1.

\section{Methods}

\section{Parasitism trials}

In order to compare abilities of $R$. insecticola 5.15 and $R$. insecticola LSR1 to confer defense, we exposed infected and uninfected control aphid sublines to a common source of $A$. ervi. For each of two $A$. pisum clones, 5A and LSR1, we used microinjection to establish two infected sublines (Oliver et al. 2003), R. insecticola 5.15 infected and $R$. insecticola LSR1 infected. All infected sublines contained stable, heritable infections, and assays for parasitoid resistance were carried out at least 15 generations after infections were established. Assays were conducted to evaluate the resistance of these aphid sublines to A. ervi, using a parasitoid stock founded by wasps collected in June 2010 in Zurich, Switzerland. In each trial, we exposed 20 aphid nymphs of similar age (48-72-h-old) on a caged plant to a single female wasp for $\sim 6 \mathrm{~h}$. We evaluated each aphid subline in 10 replicate trials, carried out in 10 randomized complete blocks. Susceptibility was quantified $10 \mathrm{~d}$ later as the proportion of individuals exposed to wasps that were mummified (i.e., in which wasps matured, killed the aphid, and pupated). We excluded four replicates because wasps escaped or died and failed to produce any mummies at all. We analyzed proportions with a generalized linear model (logit link function) in R 2.9.2 (R Development Core Team 2009), testing for the effects of block, aphid clone, subline, and the clone $\mathrm{x}$ subline interaction. Due to overdispersion, a quasibinomial error distribution with a dispersion parameter of 3.99 was used. As recommended by Crawley (2005) for quasibinomial fits, we used $F$-tests rather than $\chi^{2}$-tests for evaluating significance. Additionally, the mean development time (time from oviposition to adult emergence from mummies) of parasitoids that completed their development successfully was analyzed by ANOVA.

\section{Genomic DNA extraction and enrichment}

We extracted DNA of $R$. insecticola 5.15 from 10 ethanol-preserved M. persicae individuals of line 7.9 R5.15 (Vorburger et al. 2010) using Gentra Puregene Cell \& Tissue Kit (Qiagen) following the manufacturer's protocol. We enriched for $R$. insecticola DNA by wholegenome amplification (WGA) using a modified protocol from Pan et al. (2008) and Burke and Moran (2011). The master mix, as modified from Pan et al. (2008), consisted of $0.66 \mathrm{M}$ of Tre D-(+)-trehalose dehydrate and $1 \mathrm{uL}$ of GenomiPhi V2 enzyme mix $(1 \times 100 \mu \mathrm{L})(\mathrm{GE}$ Healthcare UK Limited). We incubated the master mix and DNA template at $30^{\circ} \mathrm{C}$ for $16 \mathrm{~h}$, followed by a $70^{\circ} \mathrm{C}$ incubation for $20 \mathrm{~min}$ to stop the reaction. We conducted two rounds of WGA. Genomic DNA was purified after each round of WGA with the DNeasy kit (Qiagen) following the manufacturer's protocol for "Cleanup of Genomic DNA." To enrich for $R$. insecticola 5.15 DNA from total genomic DNA, we took advantage of the fact that $R$. insecticola 5.15 DNA is more GC-rich than either insect or primary endosymbiont (Buchnera) DNA. We used 6-mer oligonucleotide primers (eurofins, mwg | operon) with a random mixture of $\mathrm{G}$ and $\mathrm{C}$ and with two phosphorothioate-modifed nucleotides on the 3'-end for WGA. This was successful in enriching for $R$. insecticola 5.15 DNA, and did not appear to bias coverage toward GC-rich contigs, as the correlation coefficient between depth of coverage and \% GC for contigs was low $\left(\mathrm{R}^{2}=0.068\right)$.

\section{Sequencing and annotation}

We conducted 454 FLX pyrosequencing on the DNA from WGA. The Genomics Core facility at the University of Arizona, Tucson carried out 454 library construction and sequencing. Pyrosequencing reads were assembled with Newbler (v 2.0.0), and contigs were binned using BLAST, \% GC, and read coverage as $R$. insecticola, Buchnera, pea aphid, mitochondrial, or contaminants (i.e., contigs with low coverage and no gammaproteobacterial BLAST hits). For annotation, we used two general methods. For the first method, $R$. insecticola LSR1 scaffolds were retrieved from the NCBI genbank and were used as queries in blastx searches. Unique $R$. insecticola contigs were used as queries in blastx searches of GenBank's NR protein database. The resulting set of putative ORFs was then annotated using evidence from similarity searches using BLASTP and Hmmr (Pfam_ls, TIGR-FAM8.0) (Bateman et al. 2004; Degnan et al. 2010). Our second method of genome annotation used the IMG-ER pipeline of the Joint Genome Institute (Markowitz et al. 2009). JGI's pipeline assigned genes to functional categories such as Pfam (Bateman et al. 2004), COG clusters (Tatusov et al. 1997), Kegg Ortholog terms (KO) (Kanehisa et al. 2004), Enzyme Nomenclature (Bairoch 2000), Gene Ontology terms (Gene Ontology Consortium 2004), InterPro (Mulder et al. 2005), and TIGRfam (Selengut et al. 2007). Annotations were largely consistent for the two methods, but the IMG-ER homology assignments were more conservative and identified more ORFs as encoding hypothetical proteins. Hypothetical genes $\geq 30$ amino acids that showed a significant BLASTP (E-value $<1 \times 10^{-10}$ ) hit in NCBI were reannotated based on the first method. We submitted this genome to JGI under project Id: Gi09908 and NCBI under Genome Accession: PRJNA65437; ID: 65437 using JGI's modified annotations. Homologs and unique genes of $R$. insecticola strains were identified using JGI's phylogenetic profiler tool using default settings.

\section{Phage detection}

We screened $R$. insecticola 5.15 for the presence of an APSE-like bacteriophage by conducting PCR using conserved APSE primer pairs P17/18, P23/P24, P45/51, and P35/41 (see Degnan and Moran 2008 and Supplemental Methods for PCR conditions, concentrations, and primer sequences). We also analyzed contigs from WGA for active lytic or lysogenic phage. Indicators of phage presence include large and/or high copy-number contigs encoding only phage genes.

\section{Genome Research}




\section{Phylogenetic analyses and $d_{\mathrm{N}} / d_{\mathrm{S}}$ estimates}

For the $R$. insecticola phylogeny, we retrieved homologs of three $R$. insecticola core genes ( $a c c D$, gyrB, and murE) from GenBank for five $R$. insecticola strains sampled from the aphid species $A$. pisum (strains LSR1, 8-10-1, and 5AU), Hyperomyzus lactucae, and Prociphilus fraxinifolii. We aligned nucleotide sequences with MacClade 4.06 OSX (Maddison and Maddison 2008). The three genes were concatenated into a single 1977-bp alignment, and the phylogeny and 100 nonparametric bootstraps were estimated using RAxML Blackbox 7.2.7 (Stamatakis et al. 2008) on the CIPRES Science Gateway V. 3.1 (Miller et al. 2009).

For the T1SS phylogeny, we retrieved homologs of $R$. insecticola 5.15's T1SS (genes $r t x B 2, r t x D, r t x B 1$ ) from the previously sequenced genomes of $R$. insecticola LSR1 and $H$. defensa 5 AT. Also, we obtained T1SS gene sequences from seven $H$. defensa strains that are harbored within the following whitefly and aphid hosts: Bemisia tabaci (str BTAB), Uroleucon ambrosiae (str UAMB), U. reynoldense (str UREY), U. pieloui (str UPIE), A. pisum (str A2C and A1A), and Aphis craccivora (str 5ATAC). We obtained these sequences by using PCR with degenerate primers designed from $H$. defensa 5AT (Supplemental Methods). Using degenerate primers designed from $R$. insecticola 5.15 , T1SS gene sequences were also obtained from $R$. insecticola strains from A. pisum from the United Kingdom (UK102), H. lactucae, and $P$. fraxinifolii. See Supplemental Methods for details on aphid hosts, primer sequences, PCR, and sequencing. Degenerate primers successfully amplified T1SS genes in all $H$. defensa strains. However, in some strains, not all amplicons were successfully sequenced. All partial and entire contigs of T1SS genes for $R$. insecticola and $H$. defensa strains were submitted to NCBI under accession numbers JF928377-JF928387. For all strains with complete or near complete T1SS, nucleotide sequences were aligned with MacClade 4.06 OSX (Maddison and Maddison 2008), and nongapped regions were concatenated into a single 4334-bp alignment. We conducted phylogeny estimations as above for the $R$. insecticola strain phylogeny.

We estimated pairwise estimates of nonsynonymous substitutions per nonsynonymous site $\left(d_{\mathrm{N}}\right)$ and synonymous substitutions per synonymous site $\left(d_{S}\right)$ for single-copy genes that are conserved in most gammaproteobacteria (SICO) (Lerat et al. 2003). Only SICO genes $>250$ bp that were present in both $R$. insecticola 5.15 and $R$. insecticola LSR1 were analyzed $(n=184)$. We obtained $R$. insecticola LSR1 sequences from GenBank, aligned SICO genes with MacClade 4.06 OSX (Maddison and Maddison 2008), and calculated $d_{\mathrm{N}}$ and $d_{\mathrm{S}}$ estimates using the method of Goldman and Yang (1994) in PAML (Yang 1997).

\section{Data access}

Regiella insecticola 5.15's genome is available from Joint Genome Institute's public genome database, JGI-GEBA, Gold ID: Gi09908 (http://img.jgi.doe.gov/cgi-bin/geba/main.cgi?section=TaxonDetail\& page=taxonDetail\&taxon_oid=2507262005) and NCBI. (This Whole Genome Shotgun project has been deposited at DDBJ/EMBL/ GenBank under the accession AGCA01000000. The version described in this paper is the first version, AGCA01000000.) Nucleotide data from multiple $R$. insecticola and $H$. defensa strains were submitted to the NCBI Gene Expression Omnibus (GEO) (http://www.ncbi. nlm.nih.gov/geo/) under accession numbers JF928377-JF928387.

\section{Acknowledgments}

We thank Monica Tung for help with PCR and sequencing of T1SS genes, Adam Hejmowski for his help with figures, and Brian Chang for help with data entry. We also thank Charles Godfray for kindly sending us the $R$. insecticola strain UK102 and Jenny Herzog for help with parasitism trials. We are grateful to Rahul Raghavan and
Patrick Degnan for discussions and helpful comments on the manuscript.

\section{References}

Anzaldi L, Skaar E. 2010. Overcoming the heme paradox: heme toxicity and tolerance in bacterial pathogens. Infect Immun 78: 4977-4989.

Bairoch A. 2000. The ENZYME database in 2000. Nucleic Acids Res 28: 304 305.

Baker S, Daniels C, Morona R. 1997. PhoP/Q regulated genes in Salmonella typhi: identification of melittin sensitive mutants. Microb Pathog 22: 165-179.

Bateman A, Coin L, Durbin R, Finn RD, Hollich V, Griffiths-Jones S, Khanna A, Marshall M, Moxon S, Sonnhammer ELL, et al. 2004. The Pfam protein families database. Nucleic Acids Res 32: D138-D141.

Bennett HP, Clarke DJ. 2005. The pbgPE operon in Photorhabdus luminescens is required for pathogenicity and symbiosis. J Bacteriol 187: 77-84.

Braun V, Hantke K. 2011. Recent insights into iron import by bacteria. Curr Opin Chem Biol 15: 1-7.

Brillard J, Duchaud E, Boemare N, Kunst F, Givaudan A. 2002. The Ph1A hemolysin from the entomopathogenic bacterium Photorhabdus luminescens belongs to the two-partner secretion family of hemolysins. J Bacteriol 184: 3871-3878.

Burke GR, Moran NA. 2011. Massive genomic decay in Serratia symbiotica, a recently evolved symbiont of aphids. Genome Biol Evol 3: 195-208.

Caccia S, Leonardi MG, Casartelli M, Grimaldi A, de Eguileor M, Pennacchio F, Giordana B. 2005. Nutrient absorption by Aphidius ervi larvae. J Insect Physiol 51: 1183-1192.

Coote JG. 1992. Structural and functional relationships among the RTX toxin determinants of Gram-negative bacteria. FEMS Microbiol Rev 88: 137-162.

Crawley MJ. 2005. Statistics: An introduction using R. Wiley, West Sussex, UK.

Daborn PJ, Waterfield N, Silva CP, Au CPY, Sharma S, ffrench-Constant RH. 2002. A single Photorhabdus gene, makes caterpillars floppy (mcf) allows Escherichia coli to persist within and kill insects. Proc Natl Acad Sci 99: 10742-10747.

Degnan PH, Moran NA. 2008. Diverse phage-encoded toxins in a protective insect endosymbiont. Appl Environ Microbiol 74: 6782-6791.

Degnan PH, Yu Y, Sisneros N, Wing RA, Moran NA. 2009. Hamiltonella defensa, genome evolution of a protective bacterial endosymbiont from pathogenic ancestors. Proc Natl Acad Sci 106: 9063-9068.

Degnan PH, Leonardo TE, Cass B, Hurwitz B, Stern D, Gibbs RA, Richards S, Moran NA. 2010. Dynamics of genome evolution in facultative symbionts of aphids. Environ Microbiol 12: 2060-2069.

Derzelle S, Turlin E, Duchaud E, Pages S, Kunst F, Givaudan A, Danchin A. 2004. The PhoP-PhoQ two-component regulatory system of Photorhabdus luminescens is essential for virulence in insects. J Bacteriol 186: $1270-1279$.

Edwards NJ, Monteiro MA, Faller G, Walsh EJ, Moran AP, Roberts IS, High NJ. 2000. Lewis $\mathrm{X}$ structures in the $\mathrm{O}$ antigen side-chain promote adhesion of Helicobacter pylori to the gastric epithelium. Mol Microbiol 35: 1530-1539.

Ernst RK, Yi EC, Guo L, Lim KB, Burns JL, Hackett M, Miller SI. 1999. Specific lipopolysaccharide found in cystic fibrosis airway Pseudomonas aeruginosa. Science 286: 1561-1565.

Gene Ontology Consortium. 2004. The Gene Ontology Database and Informatics Resource. Nucleic Acids Res 32: 258-261.

Gerardo NM, Altincicek B, Anselme C, Atamian H, Barribeau SM, Vos M, Duncan EJ, Evans JD, Gabaldón T, Ghanim M. 2010. Immunity and other defenses in pea aphids, Acyrthosiphon pisum. Genome Biol 11: R21. doi: 1186/gb-2010-11-2-r21.

Giordana B, Milani A, Grimaldi A, Farneti R, Casartelli M, Ambrosecchio MR, Digilio MC, Leonardi MG, de Eguileor M, Pennacchio F. 2003. Absorption of sugars and amino acids by the epidermis of Aphidius ervi larvae. J Insect Physiol 49: 1115-1124.

Goldman N, Yang Z. 1994. A codon-based model of nucleotide substitution for protein-coding DNA sequences. Mol Biol Evol 11: 725-736.

Grabenstein JP, Marceau M, Pujol C, Simonet M, Bliska JB. 2004. The response regulator PhoP of Yersinia pseudotuberculosis is important for replication in macrophages and for virulence. Infect Immun 72: 4973-4984.

Groisman E. 2001. The pleiotropic two-component regulatory system PhoPPhoQ. J Bacteriol 183: 1835-1842.

Groisman EA, Mouslim C. 2006. Sensing by bacterial regulatory systems in host and non-host environments. Nat Rev Microbiol 4: 705-709.

Groisman EA, Kayser J, Soncini FC. 1997. Regulation of polymyxin resistance and adaptation to low-Mg21 environments. J Bacteriol 179: 7040-7045.

Gross P. 1993. Insect behavioral and morphological defenses against parasitoids. Annu Rev Entomol 38: 251-273.

Guo L, Lim KB, Gunn JS, Bainbridge B, Darveau RP, Hackett M, Miller SI. 1997. Regulation of lipid A modifications by Salmonella typhimurium virulence genes phoP-phoQ. Science 276: 250-253. 
Habermann E. 1972. Bee and wasp venoms. Science 177: 314-322.

Holland B, Schmitt L, Young J. 2005. Type 1 protein secretion in bacteria, the ABC-transporter dependent pathway. Mol Membr Biol 22: 29-39.

Hueck C. 1998. Type III protein secretion systems in bacterial pathogens of animals and plants. Microbiol Mol Biol Rev 62: 379-433.

Jacques M. 1996. Role of lipo-oligosaccharides and lipopolysaccharides in bacterial adherence. Trends Microbiol 4: 408-409.

Jacques M, Paradis SE. 1998. Adhesin-receptor interactions in Pasteurellaceae. FEMS Microbiol Rev 22: 45-59.

Jimenez-Lucho VE, Joiner KA, Foulds J, Frank MM, Leive L. 1987. C3b generation is affected by the structure of the O-antigen polysaccharide in lipopolysaccharide from salmonellae. J Immunol 139: 1253-1259.

Johnson CR, Newcombe J, Thorne S, Borde HA, Eales-Reynolds LJ, Gorringe AR, Funnell SG, McFadden JJ. 2001. Generation and characterization of a PhoP homologue mutant of Neisseria meningitidis. Mol Microbiol 39: 1345-1355

Kanehisa M, Goto S, Kawashima S, Okuno Y, Hattori M. 2004. The KEGG resource for deciphering the genome. Nucleic Acids Res 32: D277-D280.

Lander ES, Waterman MS. 1988. Genomic mapping by fingerprinting random clones: a mathematical analysis. Genomics 2: 231-239.

Lerat E, Daubin V, Moran NA. 2003. From gene trees to organismal phylogeny in prokaryotes: the case of the $\gamma$-Proteobacteria. PLoS Biol 1: 101-108.

Liang-Takasaki CJ, Makela PH, Leive L. 1982. Phagocytosis of bacteria by macrophages: changing the carbohydrate of lipopolysaccharide alters interaction with complement and macrophages. J Immunol 128: 12291235 .

Liang-Takasaki CJ, Saxen H, Makela PH, Leive L. 1983. Complement activation by polysaccharide of lipopolysaccharide: an important virulence determinant of salmonellae. Infect Immun 41: 563-569.

Macfarlane ELA, Kwasnicka A, Ochs MM, Hancock REW. 1999. PhoP-PhoQ homologues in Pseudomonas aeruginosa regulate expression of the outermembrane protein OprH and polymyxin B resistance. Mol Microbiol 34: 305-316.

Maddison DR, Maddison WP. 2008. MacClade 4.06 OSX. Sinauer Associates, Inc. http://macclade.org/index.html.

Main-Hester KL, Colpitts KM, Thomas GA, Fang FC, Libby SJ. 2008. Coordinate regulation of Salmonella pathogenicity island 1 (SPI1) and SPI4 in Salmonella enterica serovar Typhimurium. Infect Immun 76: 1024-1035.

Markowitz VM, Mavromatis K, Ivanova NN, Chen IA, Chu K, Kyrpides NC. 2009. IMG ER: A System for microbial genome annotation expert review and curation. Bioinformatics 25: 2271-2278.

Matsumoto H, Young G. 2009. Translocated effectors of Yersinia. Curr Opin Microbiol 12: $94-100$.

Miller SI, Kukral AM, Mekalanos JJ. 1989. A two-component regulatory system (phoP phoQ) controls Salmonella typhimurium virulence. Proc Natl Acad Sci 86: 5054-5058.

Miller MA, Holder MT, Vos R, Midford PE, Liebowitz T, Chan L, Hoover P, Warnow T. 2009. The CIPRES Portals. CIPRES. http://www.phylo.org/ sub_sections/portal. (Archived by WebCite(r) at http:// www.webcitation.org/5imQ1JeQa).

Moran AP, Prendergast MM, Appelmelk BJ. 1996. Molecular mimicry of host structures by bacterial lipopolysaccharides and its contribution to disease. FEMS Immunol Med Microbiol 16: 105-115.

Moran NA, Degnan PH, Santos SR, Dunbar HE, Ochman H. 2005. The players in a mutualistic symbiosis: Insects, bacteria, viruses and virulence genes. Proc Natl Acad Sci 102: 16919-16926.

Moss JE, Fisher PE, Vick B, Groisman EA, Zychlinsky A. 2000. The regulatory protein PhoP controls susceptibility to the host inflammatory response in Shigella flexneri. Cell Microbiol 2: 443-452.

Mulder NJ, Apweiler R, Attwood TK, Bairoch A, Bateman A, Binns D, Bradley $\mathrm{P}$, Bork P, Bucher P, Cerutti L, et al. 2005. InterPro, progress and status in 2005. Nucleic Acids Res 33: D201-D205.

Oliver KM, Russell J, Moran NA, Hunter M. 2003. Facultative bacterial symbionts in aphids confer resistance to parasitic wasps. Proc Natl Acad Sci 100: 1803-1807.

Oliver KM, Moran NA, Hunter MS. 2005. Variation in resistance to parasitism in aphids is due to symbionts and not host genotype. Proc Natl Acad Sci 102: 12975-12800.

Oliver KM, Campos J, Moran NA, Hunter MS. 2008. Population dynamics of defensive symbionts in aphids. Proc R Soc B 275: 293-299.

Oliver KM, Degnan PH, Hunter MS, Moran NA. 2009. Bacteriophage encode factors required for protection in a symbiotic mutualism. Science $\mathbf{3 2 5}$ : 992-994.
Orth K, Xu Z, Mudgett M, Bao Z, Palmer L, Bliska J. 2000. Disruption of signaling by Yersinia effector YopJ, a ubiquitin-like protein protease. Science 290: 1594-1597.

Oyston PCF, Dorrell N, Williams K, Li S, Green M, Titball RW, Wren BW. 2000. The response regulator PhoP is important for survival under conditions of macrophage-induced stress and virulence in Yersinia pestis. Infect Immun 68: 3419-3425.

Pan X, Urban AE, Palejev D, Schulz V, Grubert F, Hu Y, Snyder M, Weissman SM. 2008. A procedure for highly specific, sensitive, and unbiased whole-genome amplification. Proc Natl Acad Sci 105: 15499-15504.

Pennacchio F, Digilio MC, Tremblay E. 1995. Biochemical and metabolic alterations in Acyrthosiphon pisum parasitized by Aphidius ervi. Arch Insect Biochem Physiol 30: 351-367.

Pennacchio F, Fanti P, Falabella P, Digilio MC, Bisaccia F, Tremblay E. 1999. Development and nutrition of the braconid wasp, Aphidius ervi in aposymbiotic host aphids. Arch Insect Biochem Physiol 40: 53-63.

Perez E, Samper S, Bordas Y, Guilhot C, Gicquel B, Martin C. 2001. An essential role for phoP in Mycobacterium tuberculosis virulence. Mol Microbiol 41: 179-187.

R Development Core Team. 2009. R: a language and environment for statistical computing. R Foundation for Statistical Computing, Vienna, Austria. http://www.R-project.org.

Rahbé Y, Digilio MC, Febvay G, Guillaud J, Fanti P, Pennacchio F. 2002. Metabolic and symbiotic interactions in amino acid pools of the pea aphid, Acyrthosiphon pisum, parasitized by the braconid Aphidius ervi. J Insect Physiol 48: 507-516.

Rietschel ET, Brade H, Holst O, Brade L, Muller-Loennies S, Mamat U, Zahringer U, Beckmann F, Seydel U, Brandenburg K, et al. 1996. Bacterial endotoxin: Chemical constitution, biological recognition, host response, and immunological detoxification. Curr Top Microbiol Immunol 216: 39-81.

Scarborough CL, Ferrari J, Godfray HCJ. 2005. Aphid protected from pathogen by endosymbiont. Science 310: 1781. doi: 10.1126/ science. 1120180 .

Selengut JD, Haft DH, Davidsen T, Ganapathy A, Gwinn-Giglio M, Nelson WC, Richter AR, White O. 2007. TIGRFAMs and genome properties: tools for the assignment of molecular function and biological process in prokaryotic genomes. Nucleic Acids Res 35: D260-D264.

Sheahan KL, Cordero CL, Satchell KJ. 2007. Autoprocessing of the Vibrio cholera RTX toxin by the cysteine protease domain. EMBO J 26: 25522561.

Stamatakis A, Hoover P, Rougemont J. 2008. A rapid bootstrap algorithm for the RAxML web-servers. Syst Biol 75: 758-771.

Tatusov RL, Koonin EV, Lipman DJ. 1997. Genomic perspective on protein families. Science 278: 631-637.

Thompson SN. 1986. Nutrition and in vitro culture of insect parasitoids. Annu Rev Entomol 31: 197-219.

Thompson J, Jones H, Perry R. 1999. Molecular characterization of the hemin uptake locus $(\mathrm{hmu})$ from Yersinia pestis and analysis of $\mathrm{hmu}$ mutants for hemin and hemoprotein utilization. Infect Immun 67: 3879-3892.

Uçkan F, Sinan S, Savasçı S, Ergin E. 2004. Determination of venom components from the endoparasitoid wasp Pimpla turionellae L. (Hymenoptera; Ichneumonidae). Ann Entomol Soc Am 97: 775-780.

Vinson SB, Iwantsch GF. 1980. Host suitability for insect parasitoids. Annu Rev Entomol 25: 397-419.

Vorburger CL, Gouskov A. 2011. Only helpful when required: A longevity cost of harbouring defensive symbionts. J Evol Biol 24: 1611-1617.

Vorburger C, Gehrer L, Rodriguez P. 2010. A strain of the bacterial symbiont Regiella insecticola protects aphids against parasitoids. Biol Lett 6: 109_ 111 .

Watson R, Millichap P, Joyce SA, Reynolds S, Clark DJ. 2010. The role of iron uptake in pathogenicity and symbiosis in Photorhabdus luminescens TT01. BMC Microbiol 10: 177. doi: 10.1186/1471-2180-10-177.

Waterman SR, Holden DW. 2003. Functions and effectors of the Salmonella pathogenicity island 2 type III secretion system. Cell Microbiol 5: 501511.

Yang Z. 1997. PAML: a program package for phylogenetic analysis by maximum likelihood. Comput Appl Biosci 13: 555-556.

Zohdy NM. 1976. On the effect of the food of Myzus persicae Sulz. on the hymenopterous parasite Aphelinus asychis Walker. Oecologia 26: 185191.

Received April 28, 2011; accepted in revised form September 6, 2011.

\section{Genome Research}

www.genome.org 


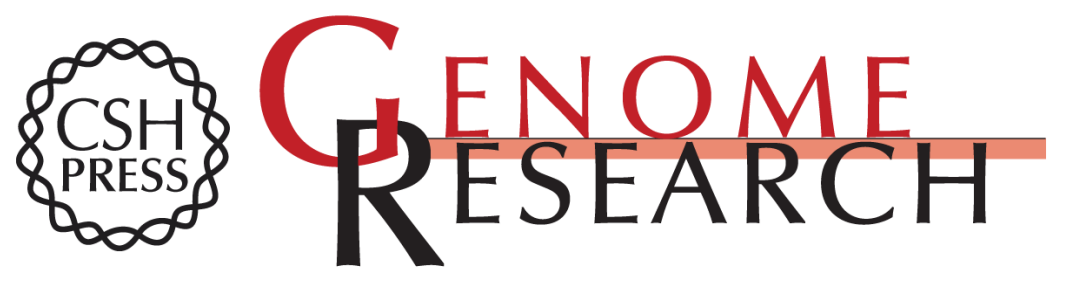

\section{Genomic basis of endosymbiont-conferred protection against an insect parasitoid}

Allison K. Hansen, Christoph Vorburger and Nancy A. Moran

Genome Res. 2012 22: 106-114 originally published online September 23, 2011

Access the most recent version at doi:10.1101/gr.125351.111

Supplemental Material

References

License

Email Alerting Service
http://genome.cshlp.org/content/suppl/2011/09/13/gr.125351.111.DC1

This article cites 75 articles, 28 of which can be accessed free at: http://genome.cshlp.org/content/22/1/106.full.html\#ref-list-1

Receive free email alerts when new articles cite this article - sign up in the box at the top right corner of the article or click here.

\section{Affordable, Accurate Sequencing.}

To subscribe to Genome Research go to:

https://genome.cshlp.org/subscriptions 